Article

\title{
On the Analyticity of Static Solutions of a Field Equation in Finsler Gravity
}

\author{
Erasmo Caponio *(D) and Antonio Masiello \\ Dipartimento di Meccanica, Matematica e Management, Politecnico di Bari, Via Orabona 4, 70125 Bari, Italy \\ * Correspondence: erasmo.caponio@poliba.it
}

Received: 29 February 2020; Accepted: 21 April 2020; Published: 22 April 2020

check for updates

\begin{abstract}
It is well-known that static vacuum solutions of Einstein equations are analytic in suitable coordinates. We ask here for an extension of this result in the context of Finsler gravity. We consider Finsler spacetimes that retain several properties of static Lorentzian spacetimes, are Berwald and have vanishing Ricci scalar.
\end{abstract}

Keywords: Finsler spacetimes; static Killing vector field; modified Einstein equation; analyticity of solutions; Berwald spaces

PACS: 04.20.Cv; 04.50.Kd

MSC: 58J60; 83C65

\section{Introduction}

In a couple of papers appeared in 1970 [1,2], H. Müller zum Hagen proved that on any $C^{3}$ static or stationary spacetime which is a vacuum solution of the Einstein equations there exists an appropriate analytic atlas such that the metric coefficients of the solution are also analytic. Our aim in this paper is to investigate if this result can be extended to static Finsler spacetimes of Berwald type. This goal forces us to analyse at least three questions:

What is the convenient definition of a Finsler spacetime?

What the one of a static Finsler spacetime?

What the field equations extending Einstein equations?

We will then consider each of the above questions in the next three sections. Section 5 will be devoted to the extension of Müller zum Hagen's result to a static Berwald spacetime.

\section{On the Definition of a Finsler Spacetime}

The idea of replacing the Lorentzian norm of a spacetime by a function positively homogeneous on the velocities goes back to G. Randers [3]. He introduced a complex-valued norm $F(x, y)=\sqrt{h_{x}(y, y)}+\omega_{x}(y)$, where $h$ is a Lorentzian metric and $\omega$ a one-form on a four-dimensional manifold $\tilde{M}$, that could take more into account the asymmetries of the physical world, in particular the "uni-direction of timelike intervals". After decades, Lorentz-Finsler norms, eventually defined only on a cone sub-bundle $\mathcal{A}$ of $T \tilde{M}$ in order to avoid complex and/or negative values, appeared again in the work of G. S. Asanov (see [4] and the references therein) about general relativity and gauge field theory. Afterwards, they have been considered 
in the study of multirefringence models in optics [5], in the classical limit of modified dispersion relations encompassing Lorentz violation in quantum gravity and in the Standard Model Extension (see, e.g., [6-10]), in studies about causality and superluminal signals (see, for example, [11,12]). Actually, Lorentz-Finsler norms had already emerged some years before the work of Asanov in a paper by H. Busemann [13], in relation to the local description of the spacetime according to an axiomatic definition called by the author timelike G-space. Inspired by [13], J.K. Beem in [14] introduced the notion of an indefinite Finsler metric as a non-reversible fiberwise positively homogeneous of degree 2 function $L=L(x, y)$, defined on the tangent bundle of $\tilde{M}$, whose fiberwise Hessian

$$
\tilde{g}(x, y)[u, v]:=\left.\frac{1}{2} \frac{\partial^{2}}{\partial s \partial t} L(x, y+s u+t v)\right|_{(s, t)=(0,0)}
$$

$u, v \in T_{x} \tilde{M}$, has index 1 for all $x \in \tilde{M}$ and $y \in T_{x} \tilde{M} \backslash\{0\}$. This definition widely extends Lorentzian geometry, with the fundamental tensor $g$ replacing the Lorentzian metric and the function

$$
F(x, \cdot):=\sqrt{|L(x, \cdot)|},
$$

giving a positively homogeneous Lorentz-Finsler norm at each tangent space $T_{x} \tilde{M}$. Notice that $F(x . \cdot)$ becomes absolutely homogeneous if $L$ is reversible, i.e., $L(x, y)=L(x,-y)$. Geodesics of $(\tilde{M}, L)$ connecting two points $p, q \in \tilde{M}$ can be defined as extremal curves of the energy functional $\gamma \in \mathcal{C}_{p q}(\tilde{M}) \mapsto \int_{0}^{1} L(\gamma, \dot{\gamma}) d s$, where $\mathcal{C}_{p q}(\tilde{M})$ is the set of all the piecewise smooth curves $\gamma:[a, b] \rightarrow \tilde{M}$ such that $\gamma(a)=p$ and $\gamma(b)=q$. It is soon realized that geodesics must satisfy the conservation law $L(\gamma, \dot{\gamma})=$ const. and, as a consequence, world-lines of freely falling particles are introduced kinematically as those timelike geodesics $(L(\gamma, \dot{\gamma})<0)$ parametrized with $L(\gamma, \dot{\gamma})=-1$. More recently, V. Perlick [15] used Beem's definition of the Finsler spacetime in order to extend Fermat's principle for light rays (i.e., geodesics satisfying $L(\gamma, \dot{\gamma})=0$ ) between a point and a light source modelled as a timelike curve. His Finslerian Fermat's principle recovers also some results that had already appeared in the study of optics in an anisotropic medium and also of sound rays in an anisotropic elastic medium (see references in [15]).

In [14], some 2-dimensional examples of indefinite Finsler metrics $L$, reversible and not, are given where the set of lightlike vectors has more than two connected components. E. Minguzzi [16] showed later that multiple light cones do not occur if $L$ is smooth on $T \tilde{M} \backslash 0$, reversible and $\operatorname{dim} \tilde{M} \geq 3$. Anyway, for non reversible and in particular for functions $L$ that are not smooth on the whole slit tangent bundle, multiple connected components are to be expected. This fact had led several authors to assume that only one of these connected components should be considered as a privileged one by the point of view of causality. The choice can be done, e.g., by prescribing a timelike, globally defined, vector field $Y$ and taking at each $x \in \tilde{M}$ the connected component which is the boundary of the set of timelike vectors containing $Y(x)$ (such as, for example, in [16,17]) or by a priori restricting $L$ to a cone sub-bundle $\mathcal{A}$ of $T \tilde{M}$, like in Asanov's definition of a Finsler norm $F$ (such as, for example, in [18-21]) or by looking only at the cone structure, without considering as fundamental the function $L$ (see [22-25]). In some physical models, anyway, indefinite Finsler metrics $L$ arise as the metrics invariant under the action of the symmetry group considered and, in general, they are defined and smooth only on an open cone sub-bundle of $T \tilde{M}$. In particular, this is the case of the Bogoslovsky metric (see, for example, [26,27]). It was observed that this is the metric that is preserved under the action of the group of transformations of the so-called Very Special Relativity [28].

Recently, a definition of a Finsler spacetime has been proposed [29] that encompasses definitions which generalize Beem's one as those in [18-20,30]. The authors declare in [29] that their definition does not include some classes of Finsler spacetimes studied in [31,32] which can be seen as generalizations 
of standard static and stationary Lorentzian spacetimes and that have already appeared in other papers [33-36]. Thus, it is worth to relax slightly the definition in [29] in order to include them.

Definition 1 (Open cone sub-bundle of $T \tilde{M})$. Let $\tilde{M}$ be a smooth connected manifold of dimension $n+1$ and $\tilde{\pi}: T \tilde{M} \rightarrow \tilde{M}$ its tangent bundle. A subset $C \subset T \tilde{M}$ will be said an open cone sub-bundle of $T \tilde{M}$, if

(i) $\tilde{\pi}(C)=\tilde{M}$;

(ii) for all $x \in \tilde{M}, C_{x}:=T_{x} \tilde{M} \cap C$ is a pointed open cone, i.e., $0 \in C_{x}, C_{x} \backslash\{0\}$ is an open subset of $T_{x} \tilde{M}$ and if $y \in C_{x}$ then $\lambda y \in C_{x}$ for each $\lambda>0$;

(iii) $C_{x}$ varies smoothly with $x \in \tilde{M}$ meaning that $C_{x} \backslash\{0\}$ is defined by the union of the solutions of a finite number of systems of inequalities in the variable $y$

$$
\left\{\begin{array}{l}
E_{1, k}(x, y)>0 \\
\cdots \\
E_{m_{k}, k}(x, y)>0
\end{array}\right.
$$

where, for each $k \in\{1, \ldots, l\}, E_{1, k}, \ldots, E_{m_{k}, k}: T \tilde{M} \rightarrow \mathbb{R}$ are $m_{k}$ smooth functions on $T \tilde{M} \backslash 0$, positively homogeneous of degree, respectively, $\alpha_{1, k}, \ldots, \alpha_{m_{k}, k}$ in $y$.

An open cone sub-bundle such that for all $x \in \tilde{M}, C_{x}$ is convex will be said a convex open cone sub-bundle. Moreover, an open cone sub-bundle without the zero section will be called a slit cone sub-bundle.

Remark 1. Notice that we do not assume that $C_{x}$ is convex nor that it is salient, i.e., that if $y \in C_{x}$ then $-y \notin C_{x}$ (indeed the open cone sub-bundle $C$ can be equal to TĨ). Being salient is instead certainly true for a convex slit cone sub-bundle. Finally, notice that (iii) implies that the boundary of a fibre of an open cone sub-bundle is the union of a finite number of piecewise smooth hypersurfaces in $T_{x} \tilde{M}$.

Definition 2 (Lorentz-Finsler metric and Finsler spacetimes). Let $C$ be an open cone sub-bundle of TM̃. A Lorentz-Finsler metric on $\tilde{M}$ is a continuous function $L: C \rightarrow \mathbb{R}$ which satisfies:

(i) $L=L(x, y)$ is fiberwise positively homogeneous of degree two, i.e., $L(x, \lambda y)=\lambda^{2} L(x, y)$, for all $x \in \tilde{M}$, $y \in C_{x}$ and all $\lambda \geq 0$

(ii) there exist a slit cone sub-bundle $A$ and an open cone sub-bundle $B$ such that $A \subset B \subset C$ and $L$ is at least $C^{1}$ on $B$ and at least $C^{4}$ on $A$ with its fundamental tensor $\tilde{g}(x, y)$, defined as in (1), being non-degenerate for all $(x, y) \in A$;

(iii) there exists a slit cone sub-bundle $T \subset A \cap L^{-1}((-\infty, 0))$ such that its closure in $L^{-1}((-\infty, 0))$, denoted by $T^{A}$, is a convex connected component of $L^{-1}((-\infty, 0))$ contained in $B$ and, for all $(x, y) \in T, \tilde{g}(x, y)$ has index 1 .

A Finsler spacetime is a smooth finite dimensional manifold $\tilde{M}$ endowed with a Lorentz-Finsler metric.

This definition differs from the one in [29] essentially because we relax the condition that there exists a connected component of $L^{-1}((-\infty, 0))$, the slit cone sub-bundle which represents all the future-pointing timelike directions physically admissible, where $L$ is smooth (and $g$ has index 1 on it). As done in [34], a quick and elegant definition of a Lorentz-Finsler metric might consist in requiring that $L$ is $C^{2}$ a.e. on $T \tilde{M}$ with fundamental tensor having index 1 a.e. on $T \tilde{M}$. Anyway, it is preferable to control the lack of smoothness of $L$, hence we allow the possibility that $\tilde{g}$ is not defined along some relevant future-pointing timelike direction where $L$ remains differentiable at least once. This requirement allows us to get geodesics at least as weak extremal contained in $B$ of the energy functional. Let us recall that a piecewise $C^{1}$ curve 
$\gamma:[a, b] \rightarrow \tilde{M}$ is a continuous curve admitting a partition $\left\{t_{i}\right\}_{i \in\{0, \ldots, m\}}, m \in \mathbb{N}$, of $[a, b]$ such that $\left.\gamma\right|_{\left[t_{i-1}, t_{i}\right]}$, for all $i \in\{1, \ldots, m\}$, is $C^{1}$. Let us denote by $\partial_{x} L: B \rightarrow T^{*} \tilde{M}$ and $\partial_{y} L: B \rightarrow T^{*} \tilde{M}$ the partial differentials of $L$ w.r.t. the first and the second variable respectively.

Definition 3 (Geodesics contained in B). Let $\gamma:[a, b] \rightarrow \tilde{M}$ be a piecewise $C^{1}$ curve such that $(\gamma, \dot{\gamma}) \subset B$ then we say that $\gamma$ is a geodesic of $(\tilde{M}, L)$ if for any piecewise $C^{1}$ vector field $\zeta$ along $\gamma$ with $\zeta(a)=\zeta(b)=0$ it holds

$$
\int_{a}^{b}\left(\partial_{x} L(\gamma, \dot{\gamma})[\zeta]+\partial_{y} L(\gamma, \dot{\gamma})[\dot{\zeta}]\right) d s=0
$$

Arguing as in [37], Prop. 2.51, it can be proved that the Legendre map of $L$ on $T^{A}$, i.e., $(x, y) \in T^{A} \mapsto$ $\partial_{y} L(x, y)[\cdot] \in T_{x}^{*} \tilde{M}$ is injective on $T^{A}$. Thus, if $\gamma:[a, b] \rightarrow \tilde{M}$ is a geodesic such that $(\gamma, \dot{\gamma}) \subset T$ then, by a standard argument about regularity of weak extremal and classical Finslerian computations (see, e.g., [38]), we get that $\gamma$ must be a $C^{3}$ curve satisfying the equation

$$
D_{\dot{\gamma}}^{\dot{\gamma}} \dot{\gamma}=0,
$$

where $D_{\dot{\gamma}}^{\dot{\gamma}}$ is the covariant derivative along $\gamma$ with reference vector $\dot{\gamma}$ defined by the Chern connection of $L$ which is well-defined on the open subset $A$ of $T \tilde{M} \backslash 0$ by (ii) of Definition 2. In local natural coordinates on $T \tilde{M}$, Equation (2) corresponds to $\ddot{\gamma}^{i}+\Gamma_{j l}^{i}(\gamma, \dot{\gamma}) \dot{\gamma}^{j} \dot{\gamma}^{l}=0$, where the components of the Chern connection are defined in Equation (9) below. If we know that the Legendre map is injective on $A$, the same result holds for all weak extremals $\gamma$ such that $(\gamma, \dot{\gamma}) \subset A$. In any case, if we know that a weak extremal $\gamma$ is $C^{1}$ and $(\gamma, \dot{\gamma}) \subset A$ then it satisfies (2) (and therefore it is actually $\left.C^{3}\right) \cdot{ }^{1}$ In particular, from (2) it follows that there exists a unique geodesic for each initial condition in $T$.

As Randers spacetime metrics show, in general $L$ will be not differentiable along null directions, i.e., along non-zero tangent vectors $(x, y)$ such that $L(x, y)=0$. In order to have a definition for lightlike geodesics of a non smooth $L$, a possible way is to require that, for every initial null conditions $(x, y)$, there exists an open maximal interval $(-\epsilon, \epsilon)$ and a $C^{1}$ curve $\gamma:(-\epsilon, \epsilon) \rightarrow \tilde{M}$, with $\gamma(0)=x$ and $\dot{\gamma}(0)=y$, such that for every sequence $\left(x_{k}, y_{k}\right) \subset T \tilde{M}$ of initial conditions of solutions $\gamma_{k}$ of (2), $\gamma$ is the limit in the $C^{1}$ topology of $\gamma_{k}$ (see [29], Def. 1-(iv), [34], Def. 1-(d)). A more general way of defining them (see [24,25]) is inspired by a well-known local property of lightlike geodesics in a spacetime:

Definition 4 (Lightlike pregeodesics). Let $N \subset T M \backslash 0$ be the set of null directions, i.e., $N:=\{(x, y) \in T \tilde{M} \backslash 0$ : $L(x, y)=0\}$. Let also $T^{N}$ be the closure of $T$ in $L^{-1}((-\infty, 0])$. A Lipschitz curve $\gamma:[a, b] \rightarrow \tilde{M}$, such that $(\gamma, \dot{\gamma}) \subset T^{N} \cap N$ a.e., is a lightlike pregeodesic if for any $s_{0} \in[a, b]$ there exists a neighbourhood $U$ of $\gamma\left(s_{0}\right)$ such that any two points in $\gamma([a, b]) \cap U$ are not connected by any Lipschitz curve $\alpha$ such that $(\alpha, \dot{\alpha}) \subset T^{A}$.

As a consequence of [25], Theorem 6.6, we have that if $T^{N} \cap N \subset A$ (i.e., $L$ is smooth on a neighbourhood of the null directions in the boundary of $T^{A}$ ) then any lightlike pregeodesics in the sense of Definition 4, is actually a geodesic, up to reparametrization, i.e., it satisfies Equation (2).

\section{About the Notion of Stationary and Static Finsler Spacetimes}

Let us recall the notion of a Killing vector field for a Finsler metric. We refer to [32] for details. Since $L$ is $C^{1}$ on the open cone sub-bundle $B$, we take $B \subset T \tilde{M} \backslash 0$ as the base space instead of the slit tangent

1 In some cases, smoothness or at least $C^{1}$-regularity of weak extremals hold; for example, this is true for some stationary splitting Finsler spacetimes and for standard static Finsler spacetimes in next section, see, respectively, [32], Prop. A2 and [31], Th. 2.13. 
bundle which is usual in Finsler geometry (compare with [39]). A vector field $K$ on $\tilde{M}$ is a Killing vector field for $(\tilde{M}, L, B)$ if $\left.K^{c}\right|_{B}(L)=0$, where $K^{c}$ denotes the complete lift of $K$ to $T \tilde{M}$ (restricted to the open subset $B)$. This is the vector on $T \tilde{M}$ whose local flow $\tilde{\psi}$ is given by $\tilde{\psi}_{t}(v)=\left(\psi_{t}(p), d \psi_{t}(p)[v]\right)$, where $\psi$ is the flow of $K, p=\tilde{\pi}(v), v \in T M$. Thus, if $K$ is a Killing vector field then $L$ is invariant under the flow of $K^{c}$. In natural local coordinates of $T \tilde{M}, K^{c}(L)$ is given by:

$$
K^{c}(L)(x, y)=K^{h}(x) \frac{\partial L}{\partial x^{h}}(x, y)+\frac{\partial K^{h}}{\partial x^{i}}(x) y^{i} \frac{\partial L}{\partial y^{h}}(x, y),
$$

for all $(x, y) \in B$ (the Einstein's sum convention is used in the above and in the following equations). It is not difficult to prove also that $K$ is a Killing field iff $\left.K^{c}\right|_{A}$ is an infinitesimal generator of local $\tilde{g}$-isometries, i.e., for each $v \in A$ and for all $v_{1}, v_{2} \in T_{\pi(v)} \tilde{M}$, we have

$$
\tilde{g}\left(\tilde{\psi}_{t}(v)\right)\left[d \psi_{t}(p)\left[v_{1}\right], d \psi_{t}(p)\left[v_{2}\right]\right]=\tilde{g}(v)\left[v_{1}, v_{2}\right]
$$

for all $t \in I_{p}$, where $I_{p} \subset \mathbb{R}$ is an interval containing 0 such that the stages $\psi_{t}$ are well-defined in a neighbourhood $U \subset \tilde{M}$ of $p=\pi(v)$ and $d \psi_{t}(p)[v] \in A$, for each $t \in I_{p}$. Thus, the Lie derivative of $\mathcal{L}_{K} \tilde{g}$ in $A$ vanishes. In local natural coordinates on $T \tilde{M}$, this amount to say that

$$
K^{c}\left(\tilde{g}_{l j}\right)+\frac{\partial K^{h}}{\partial x^{l}} \tilde{g}_{h j}+\frac{\partial K^{h}}{\partial x^{j}} \tilde{g}_{l h}=0
$$

Definition 5 (Stationary Finsler spacetime). A Finsler spacetime $(\tilde{M}, L)$ is said stationary if it is endowed with a Killing vector field $\mathrm{K}$ which is timelike, i.e., $L(x, K(x))<0$ for all $x \in \tilde{M}$.

In a Lorentzian manifold $(M, h)$, a timelike Killing vector field $K$ is said static if $\left.\operatorname{curl} K\right|_{\mathcal{D}}=0$, where $\mathcal{D}$ is the orthogonal distribution to $K$. Equivalently, $K$ is static iff $\mathcal{D}$ is locally integrable; thus, for each $p \in M$ there exist a spacelike hypersurface $S$, through $p$, orthogonal to $K$, and an open interval $I$ such that the pullback of the metric $h$ by the flow of $K$, defined in $I \times S$, is given by $-\Lambda d t^{2}+h_{0}$, where $t \in I$, $\partial_{t}$ is the pullback of $K, \Lambda=-h(K, K)$ and $h_{0}$ is the Riemannian metric induced on $S$ by $h$ (see [40], Proposition 12.38). In order to generalize this notion to Finsler spacetimes, requiring minimal regularity assumptions on the Lorentz-Finsler metric $L$, we consider $\mathcal{D}$ as the distribution of dimension $n$ on $\tilde{M}$ defined pointwise by the kernel of the one-form $\partial_{y} L(x, K(x))[\cdot]$.

Definition 6 (Static Finsler spacetime). Let $(\tilde{M}, L)$ be a stationary Finsler spacetime endowed with a timelike Killing vector field $K$, such that $(x, K(x)) \subset B$. We say that $K$ is static if $\mathcal{D}:=\operatorname{ker}\left(\partial_{y} L(x, K(x))[\cdot]\right)$ is locally integrable.

Remark 2. Let $U$ be a vector field in $\tilde{M}$ such that $(x, U(x)) \in T^{A}$, for all $x \in \tilde{M}$ and $L(x, U(x))=-1$. If the distribution $\mathcal{D}=\operatorname{ker}\left(\partial_{y} L(x, U(x))[\cdot]\right)$ is integrable then its integral manifold can be used to define the rest spaces of the observer field $U$ (see the question posed in the final paragraph of [41]). From [32], Theorem 4.8, if $K:=\sigma U$ is also a Killing vector field, for some positive function $\sigma$ on $\tilde{M}, B=T \tilde{M}$ and $L$ satisfies $L(x, K(x))=L(x,-K(x))$ and $L(x, w \pm K(x))=L(x, w)+L(x, K(x))$ for all $(x, w) \in \mathcal{D}$ then $(\tilde{M}, L)$ is locally is isometric to a standard static Finsler spacetime (see the definition below).

Recall that we have assumed that in the open cone sub-bundle $B, L$ is at least $C^{1}$, thus $\mathcal{D}$ above is well-defined. In some Finsler spacetimes, this is the best possible regularity level of $L$. Consider, for example, a type of stationary splitting Finsler spacetime introduced in [32]: assume that $\tilde{M}=\mathbb{R} \times M$ and denote with $(t, x)$ points in $\tilde{M}$ and by $(\tau, y)$ tangent vectors of $T \tilde{M}$. Let 


$$
L((t, x),(\tau, y)):=-\Lambda(x) \tau^{2}+2 b(x, y) \tau+F^{2}(x, y),
$$

where $\Lambda$ is a smooth positive function on $M, b: T M \rightarrow \mathbb{R}$ a fiberwise positively homogeneous function and $F$ a Finsler metric on $M$. Both $b$ and $F$ are assumed to be smooth on $T M \backslash 0$; moreover, the fundamental tensor $g$ of $F$ (defined as in (1) with $F^{2}$ replacing $L$ ) is positive definite for any $(x, y) \in T M \backslash 0$ while the fiberwise Hessian of $b$ (defined analogously with $b$ in place of $L$ ) is positive semi-definite for all $(x, y) \in T M \backslash 0$. Let us denote by $\mathcal{T}$ the trivial line sub-bundle of $T \tilde{M}$ defined by the vector field $\partial_{t}$. In this case $C=T \tilde{M}, B=T \tilde{M} \backslash \mathcal{T}, A=(T \tilde{M} \backslash \mathcal{T}) \cap\{((t, x),(\tau, y)) \in T \tilde{M}: \tau>0\}$ (see [32], Prop. 3.3),

$$
T=\left\{((t, x),(\tau, y)) \in T \tilde{M}: y \in T_{x} M \backslash\{0\}, \tau>\frac{b(x, y)}{\Lambda(x)}+\sqrt{\frac{b^{2}(x, y)}{\Lambda^{2}(x)}+\frac{F^{2}(x, y)}{\Lambda(x)}}\right\} \subset A
$$

and $\partial_{t}$ is timelike and Killing. A Finsler spacetime of the type (3) has been considered in [42], where it has been shown to be a solution of the field equation $R=0$ (see next section). In that paper, $L$ is a Finsler perturbation of the Schwarzschild metric, indeed $F$ is the norm of the Riemannian metric in the spacelike base and $b$ is a function conformal to the norm of the standard Riemannian metric on $S^{2}$, see Equation (40) in [42]:

$$
\begin{aligned}
& L\left((t, r, \theta, \varphi),\left(\tau, y_{r}, y_{\theta}, y_{\varphi}\right)\right):= \\
& \quad-\left(1-\frac{2 m}{r}\right) \tau^{2}+\epsilon\left(1-\frac{2 m}{r}\right) \tau \sqrt{y_{\theta}^{2}+\sin ^{2} \theta y_{\varphi}^{2}}+\frac{y_{r}^{2}}{1-\frac{2 m}{r}}+r^{2}\left(y_{\theta}^{2}+\sin ^{2} \theta y_{\varphi}^{2}\right),
\end{aligned}
$$

where $\epsilon$ is a perturbation parameter. A particular case in type (3), is when $b$ is equal to a one-form $\omega$ on $M$. In such a case, $C=B=T \tilde{M}$ (i.e., $L$ is of class $C^{1}$ on $T \tilde{M}$ ) and $A=T \tilde{M} \backslash \mathcal{T}$. The slit cone sub-bundle $T$ of timelike future-pointing vector is defined as above with $\omega$ replacing $b$; now there is also another slit cone sub-bundle associated to $L$ which is

$$
T^{-}=\left\{((t, x),(\tau, y)) \in T \tilde{M}: y \in T_{x} M \backslash\{0\}, \tau<\frac{\omega_{x}(y)}{\Lambda(x)}-\sqrt{\frac{\omega_{x}^{2}(y)}{\Lambda^{2}(x)}+\frac{F^{2}(x, y)}{\Lambda(x)}}\right\} \subset A .
$$

In particular, in this case both $T^{A}$ and $T^{-, A}$ are convex and $L$ is smooth on $N=\{((t, x),(\tau, y)) \in$ $T \tilde{M} \backslash 0: L((t, x),(\tau, y))=0\}=\left\{((t, x),(\tau, y)) \in T \tilde{M} \backslash 0: \tau=\frac{\omega_{x}(y)}{\Lambda(x)} \pm \sqrt{\frac{\omega_{x}^{2}(y)}{\Lambda^{2}(x)}+\frac{F^{2}(x, y)}{\Lambda(x)}}\right\}$. The vector field $\partial_{t}$ is a timelike Killing vector field of $(\tilde{M}, L)$ which is static if $\omega=0$ (with integral manifolds $\{t\} \times M, t \in \mathbb{R})$. Finsler spacetimes $(\mathbb{R} \times M, L)$, with $L$ of the type (3) and $\omega=0$ have been called in [31], standard static Finsler spacetime.

Remark 3. We observe that the slit cone sub-bundle $T$ is defined also as the set of timelike vectors with positive component $\tau$ of the standard static Finsler spacetime $\left(\mathbb{R} \times M, L_{\omega}\right)$, where

$$
L_{\omega}((t, x),(\tau, y)):=-\tau^{2}+F_{\omega}^{2}(x, y)
$$

and $F_{\omega}$ is given by

$$
F_{\omega}(x, y)=\frac{\omega_{x}(y)}{\Lambda(x)}+\sqrt{\frac{\omega_{x}^{2}(y)}{\Lambda^{2}(x)}+\frac{F^{2}(x, y)}{\Lambda(x)}} .
$$

In fact, from [32], Th. 5.1, $F_{\omega}$ is a Finsler metric on $M$. 


\section{Vacuum Field Equations}

In general relativity, geodesics deviation equation is used to describe the relative acceleration of a congruence of point particles. In particular, in vacuum, the absence of tidal forces implies that $R_{j i l}^{i} y^{j} y^{l}=0$, where $R_{j k l}^{i}$ are the components of the Riemann curvature tensor; as a consequence Einstein field equations $R_{j l}=R_{j i l}^{i}=0$ are satisfied and, vice versa, if $R_{j l}=0$, then $R_{j i l}^{i} y^{j} y^{l}=0$. In [42], S. Rutz used this equivalence to generalize Einstein vacuum field equations to the Finsler setting as a single scalar equation $R(x, y)=R_{i}^{i}(x, y)=0$ on the slit tangent bundle. Here $R=R(x, y)$ is the Finsler Ricci scalar defined as follows. Let $\tilde{g}^{i j}(x, y)$ the components of the inverse of the matrix representing the fundamental tensor $\tilde{g}$ at the point $(x, y) \in A$ and let $G^{i}(x, y),(x, y) \in A$, be the spray coefficients of $L$ :

$$
G^{i}(x, y):=\frac{1}{4} \tilde{g}^{i j}(x, y)\left(\frac{\partial^{2} L}{\partial x^{k} \partial y^{j}}(x, y) y^{k}-\frac{\partial L}{\partial x^{j}}(x, y)\right)
$$

so that a smooth curve $\gamma$, such that $(\gamma, \dot{\gamma}) \subset A$, is a geodesic of $L$ if and only if, in natural local coordinate on $T \tilde{M}, \ddot{\gamma}^{i}+2 G^{i}(\gamma, \dot{\gamma})=0$. Let

$$
R_{k}^{i}(x, y):=2 \frac{\partial G^{i}}{\partial x^{k}}(x, y)-y^{m} \frac{\partial^{2} G^{i}}{\partial x^{m} \partial y^{k}}(x, y)+2 G^{m}(x, y) \frac{\partial^{2} G^{i}}{\partial y^{m} \partial y^{k}}(x, y)-\frac{\partial G^{i}}{\partial y^{m}}(x, y) \frac{\partial G^{m}}{\partial y^{k}}(x, y) .
$$

The Riemann curvature of $L$ at $(x, y) \in A$ is the linear map $\mathbf{R}_{y}: T_{x} M \rightarrow T_{x} M$ given by $\mathbf{R}_{y}:=$ $R_{k}^{i}(x, y) \partial_{x^{i}} \otimes d x^{k}$. It can be shown (see [43], Equations (8.11)-(8.12)) that $R_{k}^{i}(x, y)=R_{j k l}^{i}(x, y) y^{j} y^{l}$ where $R_{j k l}^{i}$ are the components of the $h h$ part of the curvature 2-forms of the Chern connection which are equal, for any $(x, y) \in A$, to

$$
R_{j k l}^{i}(x, y):=\frac{\delta \Gamma_{j l}^{i}}{\delta x^{k}}(x, y)-\frac{\delta \Gamma_{j k}^{i}}{\delta x^{l}}(x, y)+\Gamma_{j l}^{m}(x, y) \Gamma_{m k}^{i}(x, y)-\Gamma_{j k}^{m}(x, y) \Gamma_{m l}^{i}(x, y),
$$

being $\frac{\delta}{\delta x^{i}}$ the vector field on $A$ defined by $\frac{\delta}{\delta x^{i}}:=\frac{\partial}{\partial x^{i}}-N_{i}^{m}(x, y) \frac{\partial}{\partial y^{m}}$, where $N_{i}^{m}(x, y):=\frac{\partial G^{m}}{\partial y^{i}}(x, y)$, and $\Gamma_{j k}^{i}$ are the components of the Chern connection,

$$
\Gamma_{j k}^{i}(x, y):=\frac{1}{2} \tilde{g}^{i l}(x, y)\left(\frac{\delta \tilde{g}_{l k}}{\delta x^{j}}(x, y)-\frac{\delta \tilde{g}_{j k}}{\delta x^{l}}(x, y)+\frac{\delta \tilde{g}_{l j}}{\delta x^{k}}(x, y)\right),
$$

for all $(x, y) \in A$. The Finsler Ricci scalar is then the contraction of the Riemann curvature $R(x, y):=$ $R_{i}^{i}(x, y),(x, y) \in A$. It has been observed in [29] that Rutz's equation is not variational but can be completed, in a suitable sense, to a variational equation on $A \backslash N$ (which coincides with the field equation in [44] on the set $\{(x, y) \in A: L(x, y)=-1\})$ :

$$
\frac{3 R}{L}-\frac{1}{2} \tilde{g}^{i j} \frac{\partial^{2} R}{\partial y^{i} \partial y^{j}}-\tilde{g}^{i j}\left(\frac{\delta P_{i}}{\delta x^{j}}-P_{h} \Gamma_{i j}^{h}-P_{i} P_{j}+\frac{\partial}{\partial y^{j}}\left(y^{k}\left(\frac{\delta P_{i}}{\delta x^{k}}-P_{h} \Gamma_{i k}^{h}\right)\right)\right)=0
$$

where

$$
P_{j k}^{i}:=\frac{\partial^{2} G^{i}}{\partial y^{j} \partial y^{k}}-\Gamma_{j k}^{i}
$$

are the components of the Landsberg tensor and $P_{i}=P_{l i}^{l}$. We stress that both equations $R=0$ and (10) are equivalent to Einstein vacuum equation $\operatorname{Ric}(h)=0$ if $L$ comes from a Lorentzian metric $h, L(x, y)=h_{x}(y, y)$ (see, respectively, [42], §3 and [29], §VII). 


\section{On the Analyticity of the Average Metric of a Static Berwald Solution}

We consider now a static Finsler spacetime $\tilde{M}=\mathbb{R} \times M$ with $L$ of the type (4), but $F_{\omega}$ will be any Finsler metric $F$ on $M$, not necessarily the one in (5). Let us assume also that $F$ is a Berwald metric. This means that the components of the Chern connection of $F$ (defined as in (9) with the fundamental tensor $g$ of $F$ replacing $\tilde{g})$ do not depend on $(x, y) \in T M$ or equivalently the components $N_{j}^{i}(x, y)$ are linear in $y$ (precisely, it holds $N_{j}^{i}(x, y)=\Gamma_{j k}^{i}(x) y^{k}$, see [45], Prop. 10.2.1). From (8), the components of the Riemannian curvature tensor $R_{j k l}^{i}$ of $F$ are independent of $y$ too and the Finsler Ricci scalar is equal to $R(x, y)=R_{j i l}^{i}(x) y^{j} y^{l}$ for any $(x, y) \in T M \backslash 0$.

Let us use the index 0 for the components corresponding to the coordinate $t \in \mathbb{R}$ and by $\alpha, \beta, \gamma$ the ones corresponding to coordinate systems in $M$; moreover let us distinguish Finslerian quantities of $(\tilde{M}, L)$ from the ones of $(M, F)$ by a tilde. It can be soon realized that $L$ is Berwald as well; indeed as $\tilde{g}^{00}=-1$ and $\tilde{g}^{0 \alpha}=0$, from (6), taking also into account that $L$ does not depend on $t\left(:=x^{0}\right)$ and $\frac{\partial L}{\partial x^{\alpha}}=\frac{\partial F^{2}}{\partial x^{\alpha}}$, we get $\tilde{G}^{0}=0$ and $\tilde{G}^{\alpha}((t, x),(\tau, y))=G^{\alpha}(x, y)$. Thus, $\tilde{N}_{i}^{0}=0, \tilde{N}_{0}^{\alpha}=0$ and $\tilde{N}_{\beta}^{\alpha}((t, x),(\tau, y))=N_{\beta}^{\alpha}(x, y)$, i.e., they are all linear in $(\tau, y)$.

Since $L$ is Berwald, its non-vanishing spray coefficients $\tilde{G}^{\alpha}((t, x),(\tau, y))=$ $\left.\frac{1}{2}\left(\tilde{N}_{0}^{\alpha}((t, x),(\tau, y)) \tau+\tilde{N}_{\beta}^{\alpha}((t, x),(\tau, y)) y^{\beta}\right)=\frac{1}{2} N_{\beta}^{\alpha}(x, y)\right) y^{\beta}=G^{\alpha}(x, y) y^{\beta}$ are quadratic in $y$ and then, as in [43], Prop. 7.2.2, we get $\frac{\partial^{2} \tilde{G}^{\alpha}}{\partial y^{i} \beta \partial y^{k}}=\tilde{\Gamma}_{i k}^{\alpha}$. Since $\tilde{g}^{00}=\tilde{g}_{00}=-1, \tilde{g}^{0, \alpha}=\tilde{g}_{0 \alpha}=0$ and $\frac{\delta \tilde{g}_{j k}}{\delta x^{0}}=0$, for all $j, k \in\{0, \ldots n\}$, we also have that $\tilde{\Gamma}_{j k}^{0}=0$, for all $j, k \in\{0, \ldots n\}$. Thus, the Landsberg tensor $\tilde{P}_{j k}^{i}$ vanishes. Hence, for Berwald $L,(10)$ reduces to

$$
\frac{3 \tilde{R}}{L}-\frac{1}{2} \tilde{g}^{i j} \frac{\partial^{2} \tilde{R}}{\partial y^{i} \partial y^{j}}=0
$$

Taking into account that $\frac{\partial^{2} \tilde{G}^{\alpha}}{\partial y^{0} \partial y^{k}}=\frac{\partial^{2} G^{\alpha}}{\partial y^{0} \partial y^{k}}=0$, for all $k \in\{0, \ldots, n\}$, this also implies that $\tilde{\Gamma}_{0 k}^{\alpha}=0$ and $\tilde{\Gamma}_{\beta \gamma}^{\alpha}(t, x)=\Gamma_{\beta \gamma}^{\alpha}(x)$, that could be also proved directly by (9). Thus, $\tilde{R}_{j 0 l}^{0}=0, \tilde{R}_{0 \alpha l}^{\alpha}=0=\tilde{R}_{j \alpha 0}^{\alpha}$ and $\tilde{R}_{\beta \alpha \gamma}^{\alpha}((t, x),(\tau, y))=\tilde{R}_{\beta \alpha \gamma}^{\alpha}(t, x)=R_{\beta \alpha \gamma}^{\alpha}(x)$, which imply that the Finsler Ricci scalar of $F$ and $L$ coincide. Thus, if $F$, or equivalently $L$, has vanishing Ricci scalar, $0=R(x, y)=\tilde{R}((t, x),(\tau, y))$ for all $((t, x),(\tau, y)) \in T \tilde{M} \backslash 0$ then $L$ satisfies Equation $(10)$ on $T \tilde{M} \backslash(\mathcal{T} \cup N){ }^{2}$

It is well-known that the components (9) of the Chern connection of a Berwald metric can be obtained from different Riemannian metrics as their Christoffel symbols, [47]. In particular, see [48], this is true for the Riemannian metric

$$
h_{x}\left(V_{1}, V_{2}\right):=\frac{\int_{S_{x}} g(x, y)\left[V_{1}, V_{2}\right] d \lambda(y)}{\int_{S_{x}} d \lambda(y)}
$$

where $S_{x}:=\left\{y \in T_{x} M: F(y)=1\right\}, x \in M$ and $d \lambda(y)$ is the measure induced on $S_{x}$ by the Lebesgue measure on $\mathbb{R}^{n}$.

Let us assume that $F$ is of class $C^{4}$ on $T M \backslash 0$; then $g(x, y)$ is of class $C^{2}$ on $T M \backslash 0$. Notice that the indicatrix bundle $\{(x, y) \in T M: F(x, y)=1\}$ is a $C^{4}$ embedded hypersurface in $T M$. Thus, both the area of $S_{x}$ and the numerator in (11) are $C^{2}$ in $x$ and then $h$ is a $C^{2}$ Riemannian metric on M. From (8) and the fact that $F$ is Berwald, the components $R_{j k l}^{i}$ are equal to the ones of the Riemannian curvature tensor of $h$ and then we have

2 The converse is true only in some cases, for example for some Bogoslowski-Berwald metric, see [46]. 


$$
\operatorname{Ric}(h)_{\alpha \beta}(x)=R_{\alpha m \beta}^{m}(x)=\frac{1}{2} \frac{\partial^{2}}{\partial y^{\alpha} \partial y^{\beta}}\left(R_{j m l}^{m}(x) y^{j} y^{l}\right)=\frac{1}{2} \frac{\partial^{2} R}{\partial y^{\alpha} \partial y^{\beta}}(x, y)=0 .
$$

From [49], Theorem 4.5, it follows that in an atlas of $M$ of harmonic coordinates of $h, h$ itself is analytic. We can summarize the above reasoning in the following result:

Theorem 1. Let $(\tilde{M}, L)$ be a standard static Finsler spacetime, $\tilde{M}=\mathbb{R} \times M, L((t, x),(\tau, y))=-t^{2}+F^{2}(x, y)$. Assume that $F \in C^{4}(T M \backslash 0)$ is Berwald with vanishing Ricci Finsler scalar $R$, then $L$ is Berwald, satisfies the field Equation (10) with and the metric $\frac{\int_{S_{x}} g(x, y)[\cdot, \cdot] d \lambda(y)}{\int_{S_{x}} d \lambda(y)}$ on $M$, where $g$ is the fundamental tensor of the Finsler metric $F$, is analytic in its harmonic coordinates.

Let us now consider the case when $F$ is equal to $F_{\omega}$ in (5); in light of Theorem 1 we would like to have conditions ensuring that $F_{\omega}$ is Berwald. It is well-known that for a Randers metric $F=\alpha+\beta$, where $\alpha$ is the norm of a Riemannian metric and $\beta$ a one-form, this holds if and only if $\nabla \beta=0$, where $\nabla$ is the Levi-Civita connection of the Riemannian metric (see ([45] Th. 11.5.1)). Let us see that a sufficient condition of this type holds for $F_{\omega}$ as well. Let us denote by $\beta$ the one-form on $M$ defined as $\beta:=\omega / \Lambda$ and by $G$ the Finsler metric given by $G:=\left(F^{2} / \Lambda+\beta^{2}\right)^{1 / 2}$.

Proposition 1. Assume that the Finsler metric $F / \sqrt{\Lambda}$ on $M$ is Berwald with vanishing Ricci scalar and that $D \beta=0$, where $D$ is the linear covariant derivative on $M$ induced by the Chern connection of $F / \sqrt{\Lambda}$. Then the Finsler metric $G+\beta$ is Berwald with vanishing Ricci scalar as well.

Proof. Let us show firstly that $G$ is Berwald. In order to evaluate the spray coefficients of $G$, we compute the geodesics equation of the Finsler manifold $(M, G)$ as the Euler-Lagrange equation of the energy functional $E_{G}$ of $G$. Without loosing generality, we can assume for this purpose that $\gamma:[a, b] \rightarrow M$, $\gamma=\gamma(s)$, is a smooth regular curve (i.e., $\dot{\gamma}(s) \neq 0$, for all $s \in[a, b]$ ) and that $\sigma:[-\epsilon, \epsilon] \times[a, b] \rightarrow M$, $\sigma=\sigma(r, s)$, is a smooth variation of $\gamma$ (i.e., $\sigma(0, \cdot)=\gamma$ ) such that for all $r \in[-\epsilon, \epsilon], \sigma(r, a)=\gamma(a)$, $\sigma(r, b)=\gamma(b)$ and $\sigma(r, \cdot)$ is regular as well. Let us denote respectively by $T$ and $U$ the vector field along $\sigma$ defined by $\partial_{t} \sigma$ and $\partial_{r} \sigma$. Now, in order to compute the variation of $E_{G}$ associated to $\sigma$, we can consider separately the terms coming from the variation of the energy functional of $F / \sqrt{\Lambda}$ and the ones coming from the variation of $\frac{1}{2} \int_{a}^{b} \beta^{2}(\dot{\gamma}) d s$. The variation of the latter functional is equal to

$$
\frac{1}{2} \int_{a}^{b} \partial_{r}\left(\beta^{2}(T)\right) d s=\int_{a}^{b} \beta(T)\left(\left(D_{U} \beta\right)(T)+\beta\left(D_{U} T\right)\right) d s=\int_{a}^{b} \beta(T) \beta\left(D_{T} U\right) d s
$$

where we have used the fact that the connection $D$ is torsion free (see ([45] p. 262)) and hence $D_{U} T=D_{T} U$. Evaluating (12) at $r=0$ gives

$$
\int_{a}^{b} \beta(\dot{\gamma}) \beta\left(D_{\dot{\gamma}} V\right) d s=\int_{a}^{b} \beta(\dot{\gamma}) \frac{d}{d s} \beta(V) d s=-\int_{a}^{b} \beta(V) \frac{d}{d s} \beta(\dot{\gamma}) d s=-\int_{a}^{b} g(\gamma, \dot{\gamma})[B, V] \frac{d}{d s} \beta(\dot{\gamma}) d s,
$$

where $V$ is the variational vector field associated to $\sigma$, i.e., $V:=U(\sigma(0, \cdot))=\left.\partial_{r} \sigma(r, \cdot)\right|_{r=0}$ and $B$ is the vector field along $\gamma$ representing $\beta$ with respect to the Riemannian metric, over $\gamma, g(\gamma, \dot{\gamma})[\cdot, \cdot], g$ being the fundamental tensor of $F / \sqrt{\Lambda}$. As the variation of the energy functional of $F / \sqrt{\Lambda}$ at $r=0$ gives $\int_{a}^{b} g(\gamma, \dot{\gamma})\left[\dot{\gamma}, D_{\dot{\gamma}} V\right] d s$, we get that a a smooth critical point $\gamma$ of $E_{G}$ satisfies the equation

$$
D_{\dot{\gamma}} \dot{\gamma}+B \frac{d}{d s} \beta(\dot{\gamma})=0
$$


Hence

$$
0=\beta\left(D_{\dot{\gamma}} \dot{\gamma}\right)+\beta(B) \frac{d}{d s} \beta(\dot{\gamma})=\frac{d}{d s} \beta(\dot{\gamma})+\beta(B) \frac{d}{d s} \beta(\dot{\gamma})=\frac{d}{d s} \beta(\dot{\gamma})(1+\beta(B))
$$

As $\beta(B)=g(\gamma, \dot{\gamma})[B, B] \geq 0$, we get $\frac{d}{d s} \beta(\dot{\gamma})=0$ and hence $\gamma$ satisfy the equation $D_{\dot{\gamma}} \dot{\gamma}=0$. This implies that the spray coefficients of $G$ are quadratic in the velocities and then $G$ is Berwald. Let us now prove that also $G+\beta$ is Berwald. To this end, let us compute the variation of the length functional $\ell$ of $G+\beta$. As above, let us consider a smooth regular curve $\gamma$. Since $\ell$ is invariant under orienting preserving reparametrization, we can assume that $\gamma$ is parametrized w.r.t the arch length of $G$, i.e., $G(\gamma, \dot{\gamma})=1$. Let $l$ be the length of $\gamma$ w.r.t. G. Arguing as above, the first variation of $\ell$ at $r=0$ is equal to

$$
\int_{0}^{l} \beta\left(D_{\gamma} V\right) d s+\int_{0}^{l}\left(g(\gamma, \dot{\gamma})\left[\dot{\gamma}, D_{\dot{\gamma}} V\right]-g(\gamma, \dot{\gamma})[B, V] \frac{d}{d s} \beta(\dot{\gamma})\right) d s .
$$

The first integral above is equal to $\int_{0}^{l} \frac{d}{d s} \beta(V) d s$ and hence it vanishes for all variational vector fields $V$. Therefore, the critical points of $\ell$ parametrized w.r.t. to the arc length of $G$ satisfies (13) and then, as above, they do satisfy equation $D_{\dot{\gamma}} \dot{\gamma}=0$. Since $\frac{d}{d s} \beta(\dot{\gamma})=0, \beta(\dot{\gamma})=$ const., i.e., $\gamma$ is also affinely parametrized for the metric $G+\beta$, hence it is a geodesic of this metric. This implies that $D_{\dot{\gamma}} \dot{\gamma}=0$ is also the geodesics equation of $G+\beta$ and the spray coefficients of this metric are equal to the ones of $F / \sqrt{\Lambda}$. As a consequence, $G+\beta$ is Berwald and its Finsler Ricci scalar vanishes because it is equal to the Finsler Ricci scalar of $F / \sqrt{\Lambda}$ (recall (7)).

Remark 4. A similar proof shows that $D \beta=0$ is also a sufficient condition for a Randers variation of a Finsler metric $F$ of Berwald type (i.e., for a Finsler metric of the type $F+\beta$ with $F(x, y)+\beta_{x}(y)>0$ for all $(x, y) \in T M \backslash 0$ ) to be Berwald as well. This extends beyond the case that F is Riemannian the sufficient condition in [45], Th. 11.5.1.

\section{Conclusions}

We have reviewed the mathematical definitions of a Finsler spacetime and of a static timelike Killing vector field on it, based on a fundamental function $L$ with low regularity assumptions. In particular, we have relaxed the requirement in [29] about smoothness of $L$ on the open cone sub-bundle defining admissible timelike future-oriented vectors, in order to include static and stationary Finsler spacetimes that split as a product $\mathbb{R} \times M$. We have then considered Berwald static Finsler spacetime under the point of view of the analyticity of the solutions of the Rutz's equation $\tilde{R}(x, y)=0$ (and then satisfying also the field Equation (10) proposed in [29]). We have obtained a partial result in this direction stating analyticity (in its harmonic coordinates) of any Riemannian metric whose Levi-Civita connection coincide with the Chern connection of the Finsler metric on the base $M$. In particular, this holds for the metric (11) obtained as an average of the fundamental tensor of the Finsler metric on the base $M$.

The existence of analytic solutions (in a fixed coordinate system) of the Rutz's equation has been recently obtained for Berwald Finsler pp-waves in [50] introduced there (see also [51], §4). The Berwald static case that we have considered is, on the other hand, dynamical equivalent to the classical Lorentzian static case, at least when the dynamic is governed by the Rutz's equation. Nevertheless, extending Theorem 1 to more general classes of Finsler function $F$ seems difficult due to the lack of ellipticity and quasi-diagonality of the system of equations $R_{\alpha \beta}=R_{\alpha l \beta}^{l}=0$ (that could be considered instead of the scalar equation $R=0$, see [42], §3), even writing it in harmonic coordinates w.r.t. the horizontal Laplacian of $F$, see [52], Remark 5. From this point of view, it might be interesting to analyse a generalization of the Einstein field equations on the whole tangent bundle of the spacetime, obtained recently [53], based on Sasaki type metrics and nonlinear connections on it. 
Author Contributions: All authors have equally contributed, read and agreed to the published version of the manuscript.

Funding: The authors are supported by PRIN 2017JPCAPN Qualitative and quantitative aspects of nonlinear PDEs.

Acknowledgments: We would like to thank both reviewers for their comments that helped us to improve the quality of the paper.

Conflicts of Interest: The authors declare no conflict of interest.

\section{References}

1. Müller zum Hagen, $\mathrm{H}$. On the analyticity of static vacuum solutions of Einstein's equations. Proc. Camb. Philos. Soc. 1970, 67, 415-421. [CrossRef]

2. Müller zum Hagen, H. On the analyticity of stationary vacuum solutions of Einstein's equation. Proc. Camb. Philos. Soc. 1970, 68, 199-201. [CrossRef]

3. Randers, G. On an asymmetrical metric in the fourspace of General Relativity. Phys. Rev. 1941, 59, 195-199. [CrossRef]

4. Asanov, G.S. Finsler Geometry, Relativity and Gauge Theories; D. Reidel Publishing Co.: Dordrecht, The Netherlands, 1985.

5. Skakala, J.; Visser, M. Bi-metric pseudo-Finslerian spacetimes. J. Geom. Phys. 2011, 61, 1396-1400. [CrossRef]

6. Girelli, F.; Liberati, S.; Sindoni, L. Planck-scale modified dispersion relations and Finsler geometry. Phys. Rev. D 2007, 75, 064015. [CrossRef]

7. Vacaru, S.I. Finsler branes and quantum gravity phenomenology with Lorentz symmetry violations. Class. Quantum Grav. 2011, 28, 215001. [CrossRef]

8. Kostelecký, V.A.; Russell, N.; Tso, R. Bipartite Riemann-Finsler geometry and Lorentz violation. Phys. Lett. B 2012, 716, 470-474. [CrossRef]

9. Russell, N. Finsler-like structures from Lorentz-breaking classical particles. Phys. Rev. D 2015, 91, 045008. [CrossRef]

10. Colladay, D. Extended hamiltonian formalism and Lorentz-violating lagrangians. Phys. Lett. B 2017, 772, $694-698$. [CrossRef]

11. Chang, Z.; Li, X.; Wang, S. Opera Superluminal Neutrinos and Kinematics in Finsler Spacetime. Mod. Phys. Lett. A 2012, 27, 1250058. [CrossRef]

12. Pfeifer, C.; Wohlfarth, M.N.R. Beyond the speed of light on Finsler spacetimes. Phys. Lett. B 2012, 712, $284-288$. [CrossRef]

13. Busemann, H. Timelike spaces. Diss. Math. Rozprawy Mat. 1967, 53, 52.

14. Beem, J.K. Indefinite Finsler spaces and timelike spaces. Canad. J. Math. 1970, 22, 1035-1039. [CrossRef]

15. Perlick, V. Fermat principle in Finsler spacetimes. Gen. Relativ. Gravit. 2006, 38, 365-380. [CrossRef]

16. Minguzzi, E. Light cones in Finsler spacetime. Commun. Math. Phys. 2015, 334, 1529-1551. [CrossRef]

17. Gallego Torromé, R.; Piccione, P.; Vitório, H. On Fermat's principle for causal curves in time oriented Finsler spacetimes. J. Math. Phys. 2012, 53, 123511. [CrossRef]

18. Javaloyes, M.A.; Sánchez, M. Finsler metrics and relativistic spacetimes. Int. J. Geom. Methods Mod. Phys. 2014, 11, 1460032. [CrossRef]

19. Aazami, A.B.; Javaloyes, M.A. Penrose's singularity theorem in a Finsler spacetime. Class. Quantum Grav. 2016, 33, 025003. [CrossRef]

20. Minguzzi, E. Affine sphere relativity. Commun. Math. Phys. 2017, 350, 749-801. [CrossRef]

21. Minguzzi, E. Affine sphere spacetimes which satisfy the relativity principle. Phys. Rev. D 2017, 95, 024019. [CrossRef]

22. Fathi, A. Time functions revisited. Int. J. Geom. Methods Mod. Phys. 2015, 12, 1560027. [CrossRef]

23. Bernard, P.; Suhr, S. Lyapounov functions of closed cone fields: From Conley theory to time functions. Commun. Math. Phys. 2018, 359, 467-498. [CrossRef] 
24. Minguzzi, E. Causality theory for closed cone structures with applications. Rev. Math. Phys. 2019, 31, 1930001. [CrossRef]

25. Javaloyes, M.A.; Sánchez, M. On the definition and examples of cones and Finsler spacetimes. Rev. R. Acad. Cienc. Exactas Fís. Nat. Ser. A Mat. RACSAM 2020, 114, 30. [CrossRef]

26. Bogoslovsky, G.Y.; Goenner, H.F. Finslerian spaces possessing local relativistic symmetry. Gen. Relativ. Gravit. 1999, 31, 1565-1603. [CrossRef]

27. Kouretsis, A.P.; Stathakopoulos, M.; Stavrinos, P.C. General very special relativity in Finsler cosmology. Phys. Rev. D 2009, 79, 104011. [CrossRef]

28. Gibbons, G.W.; Gomis, J.; Pope, C.N. General very special relativity is Finsler geometry. Phys. Rev. D 2007, 76, 081701. [CrossRef]

29. Hohmann, M.; Pfeifer, C.; Voicu, N. Finsler gravity action from variational completion. Phys. Rev. D 2019, 100, 064035. [CrossRef]

30. Pfeifer, C.; Wohlfarth, M.N.R. Causal structure and electrodynamics on Finsler spacetimes. Phys. Rev. D 2011, 84, 044039. [CrossRef]

31. Caponio, E.; Stancarone, G. Standard static Finsler spacetimes. Int. J. Geom. Methods Mod. Phys. 2016, 13, 1650040. [CrossRef]

32. Caponio, E.; Stancarone, G. On Finsler spacetimes with a timelike Killing vector field. Class. Quantum Grav. 2018, 35, 085007. [CrossRef]

33. Asanov, G.S. Finslerian metric functions over the product $R \times M$ and their potential applications. Rep. Math. Phys. 1998, 41, 117-132. [CrossRef]

34. Lämmerzahl, C.; Perlick, V.; Hasse, W. Observable effects in a class of spherically symmetric static Finsler spacetimes. Phys. Rev. D 2012, 86, 104042. [CrossRef]

35. Skákala, J.; Visser, M. Pseudo-Finslerian Space-Times and Multirefringence. Int. J. Mod. Phys. D 2010, 19, 1119-1146. [CrossRef]

36. Li, X.; Chang, Z. Exact solution of vacuum field equation in Finsler spacetime. Phys. Rev. D 2014, 90, 064049. [CrossRef]

37. Caponio, E.; Javaloyes, M.A.; Sánchez, M. Wind Finslerian structures: From Zermelo's navigation to the causality of spacetimes. arXiv 2017, arXiv:1407.5494v5.

38. Caponio, E.; Javaloyes, M.A.; Masiello, A. On the energy functional on Finsler manifolds and applications to stationary spacetimes. Math. Ann. 2011, 351, 365-392. [CrossRef]

39. Lovas, R.L. On the Killing vector fields of generalized metrics. SUT J. Math. 2004, 40, 133-156.

40. O'Neill, B. Semi-Riemannian Geometry; Academic Press Inc.: New York, NY, USA, 1983.

41. Pfeifer, C. Finsler spacetime geometry in physics. Int. J. Geom. Methods Mod. Phys. 2019, 16, 1941004. [CrossRef]

42. Rutz, S.F. A Finsler generalisation of Einstein's vacuum field equations. Gen. Relativ. Gravit. 1993, 25, 1139-1158. [CrossRef]

43. Shen, Z. Lectures on Finsler Geometry; World Scientific Publishing Co.: Singapore, 2001.

44. Pfeifer, C.; Wohlfarth, M.N.R. Finsler geometric extension of Einstein gravity. Phys. Rev. D 2012, 85, 064009. [CrossRef]

45. Bao, D.; Chern, S.S.; Shen, Z. An Introduction to Riemann-Finsler Geometry; Springer: New York, NY, USA, 2000.

46. Fuster, A.; Pabst, C.; Pfeifer, C. Berwald spacetimes and very special relativity. Phys. Rev. D 2018, 98, 084062. [CrossRef]

47. Szabó, Z.I. Positive definite Berwald spaces. Structure theorems on Berwald spaces. Tensor. New Ser. 1981, 35, 25-39.

48. Crampin, M. On the construction of Riemannian metrics for Berwald spaces by averaging. Houst. J. Math. 2014, 40, 737-750.

49. DeTurck, D.M.; Kazdan, J.L. Some regularity theorems in Riemannian geometry. Ann. Scient. L'École Norm. Sup. 1981, 14, 249-260. [CrossRef]

50. Fuster, A.; Pabst, C.U. Finsler pp-waves. Phys. Rev. D 2016, 94, 104072. [CrossRef] 
51. Gómez-Lobo, A.G.P.; Minguzzi, E. Pseudo-Finsler Spaces Modeled on a Pseudo-Minkowski Space. Rep. Math. Phys. 2018, 82, 29-42. [CrossRef]

52. Caponio, E.; Masiello, A. Harmonic Coordinates for the Nonlinear Finsler Laplacian and Some Regularity Results for Berwald Metrics. Axioms 2019, 8, 83. [CrossRef]

53. Triantafyllopoulos, A; Stavrinos, P. C. Weak field equations and generalized FRW cosmology on the tangent Lorentz bundle. Class. Quantum Grav. 2018, 35, 085011.[CrossRef]

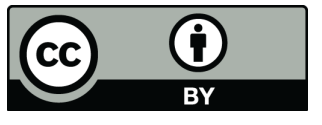

(C) 2020 by the authors. Licensee MDPI, Basel, Switzerland. This article is an open access article distributed under the terms and conditions of the Creative Commons Attribution (CC BY) license (http:/ / creativecommons.org/licenses/by/4.0/). 